\author{
Military Technical College \\ Kobry Elkobbah, \\ Cairo, Egypt
}

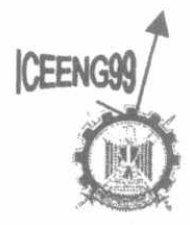

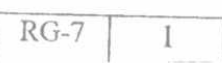

$2^{\text {nd }}$ International Conference

on Electrical Engineering

ICEENG 1999

\title{
FUSING AND ARMING SYSTEMS FOR SURFACE-TO-AIR MISSILES
}

\author{
Khaled G. Aly*, and Mohsen S. Aly**
}

\begin{abstract}
The efficiency of the anti-aircraft guided missile does not depend only upon the correct choice of the guidance and control systems, but also upon the correct choice of the warhead explosion instant. Large projectiles and all surface-to-air missiles carry warheads. There are many types of warheads. The fragmentation type is usually used in the surface-to-air missiles. A fuse is required in all projectiles and missiles equipped with warheads. There are different types of proximity fuses, but the radio fuse type is almost usually used for the anti-aircraft missiles. It reacts on the reflected radio signals from the engaged target and makes the warhead explodes within the effective distance from the target.

The optimum operation of the fusing system results in maximum probability of target destruction. That situation is achieved by the complete coincidence between the fuse activation zone and the target destruction zone. The radio fuse activation zone is determined by the fuse antenna radiation pattern. However, the target destruction zone is determined by the relative velocities of the fragments with respect to the target velocity in the space. This coincidence is verified by different techniques as varying the initiation instant of the warhead, moving the radio fuse antenna radiation pattern in the space, changing the pyrotechnic exploder position on the warhead detonation tube, and rotating the warhead around an axis perpendicular to the missile longitudinal axis.

In this paper, the variation of the explosion instant of the warhead is discussed. A computational algorithm is developed to solve the mathematical model of a typical antiaircraft radio fusing and arming systems. A computer code written with $\mathrm{C}$ language is then built to obtain numerical results. The number of fragments that hit the target, which is taken as a measure to the kill probability, is calculated for various engagement geometries. The effect of the final miss distance and missile and target velocities on the destruction probability level is computed. Results show that with a RC integrating circuit being employed as a decision circuit, reasonable destruction probability can be achieved for a wide variety of engagement scenarios. However better results can be achieved with more intelligent circuits being employed.
\end{abstract}

Keywords: Guidance and Control, Radar, and Radio Fuse.

* Ph.D. student, Syrian Army

** Ass. Prof., Radar and Guidance Dpt., Military Technical College, Cairo, Egypt 


\section{1-INTRODUCTION}

The efficiency of the anti-aircraft guided missile does not depend only upon the correct choice of the guidance and control systems, but also upon the correct choice of the warhead explosion instant. Large projectiles and all surface-to-air missiles carry warheads. There are many types of warheads. The fragmentation type is usually used in the surface-to-air missiles. A fuse is required in all projectiles and missiles equipped with warheads. There are different types of proximity fuses, but the radio fuse type is almost usually used for the anti-aircraft missiles. It reacts on the reflected radio signals from the engaged target and makes the warhead explodes within the effective distance from the target [1].

In this paper a computational algorithm is developed for the calculation of the number of fragments that may hit the target for a command guided missile equipped with a continuous wave Doppler principle radio fuse and a fragmentation type warhead. A computer code written with Borland $\mathrm{C}$ is then constructed to obtain numerical results. It is noted that the parameters that affect the number of fragments that hit the engaged target are numerous. Most of these parameters depend on the engagement geometry near the end of the missile flight mission. This engagement geometry depends on the guidance system, and the target flight behavior [2].

In this work, many engagements scenarios and straight target trajectories are considered to carry out the evaluation of the fuse system efficiency. In each case, the number of fragments that may hit the target is calculated. The reason for considering straight target trajectory is that the process of target detection and warhead explosions takes few milliseconds. During this small time, the target trajectory can be fairly approximated by straight line; even if the target maneuvers. The effect of missile-target miss distance is first considered. Then the effect of engagement direction is discussed. Analyzing the effect of the emitted fragment speed follows this. Results show that with a RC integrating circuit (employed as a decision circuit) reasonable fragments that hit the target can be achieved for a wide variety of engagement scenarios. However, better results can be achieved with more intelligent circuits being employed.

\section{2-CHARACTERISTICS OF THE UNDERLYING ARMING AND FUSING SYSTEMS}

The basic parameters of the underlying arming and fusing systems are as follows:

a. Number of fragments generated by the explosion, expressed empirically, by [4], as:

$N=\mu \frac{q_{e} \cdot \sigma \cdot x^{2}}{D} \frac{\chi^{2}+.5}{\chi^{2}-1}$

Where

$\mathrm{N}$ is the total number of fragments,

$\mu$ is the coefficient expressing the influence of explosive material,

$D$ is the caliber of warhead [cm],

$\mathrm{q}_{\mathrm{e}}$ is the weight of high explosive charge $[\mathrm{g}]$,

$\sigma_{e}$ is the elastic limit of warhead body material $\left[\mathrm{kg} / \mathrm{mm}^{2}\right]$,

$\sigma$ is the strength limit of warhead body $\left[\mathrm{kg} / \mathrm{mm}^{2}\right]$

$\delta$ is the specific elongation of body material [\%], and 
$\chi$ is the constructional coefficient expressing the fragmentation ability of warhead.

b. Speed of the fragment, the additional speed of the fragment $\left(V_{d}\right)$ is imparted to the fragment upon exploding the charge and is determined by [3], as:

$$
V_{d}=0.353 D_{1} \sqrt{\frac{3 \beta_{e} f \lambda_{1}}{3+\beta_{e} f}} \quad \text { with } \beta_{e}>0.5
$$

Where

$D_{1}$ is the burning speed of the explosive and is in the order of $7000 \div 8000 \mathrm{~m} / \mathrm{sec}$,

$\lambda_{1}$ is determined by [1], as:

$\lambda_{1}=\left\{\begin{array}{lll}0.75 & \text { with } & Q<10 \mathrm{k} . \mathrm{g} \\ 0.75 \div 1 & \text { with } & 10 \leq Q \leq 100 \mathrm{k} . \mathrm{g} \\ 1.0 & \text { with } & Q>100 \mathrm{k.g}\end{array}\right\}$

$f$ is a coefficient depending on the dimensions and shape of the warhead, $Q$ is the total weight of warhead, and $\beta_{e}$ is the warhead factor influencing the speed of the fragments upon explosion.

The initial speed of the fragment $V_{i}$ is the geometric summation of the additional speed of the fragment and the missile speed. Upon explosion, the fragments are released with initial velocity $\mathrm{V}_{\mathbf{i}}$. However, their velocity decreases exponentially by time as [1]:

$V_{R}=V_{i}^{-K R}$

Where $\mathrm{R}$ is the traveled distance by the fragments, and $\mathrm{K}$ is the ballistic coefficient determining the influence of the air density, shape and weight of the fragment, and the influence of dynamic pressure upon the speed of the fragment.

c. Warhead static destruction zone, when the warhead bursts the fragments disperses at a certain zone called warhead static destruction zone, which is around 5-30 degrees (in the present case). The axis of static emission angle may be perpendicular to the warhead roll axis or may be inclined forward or backward at a definite angle not more than 15 degrees depending on the mode of the warhead initiation or the position of the pyrotechnic igniter.

d. Warhead dynamic destruction zone, the central axis of the dynamic dispersion zone coincides with the initial velocity of the fragment, as shown in Fig. 1 [1].

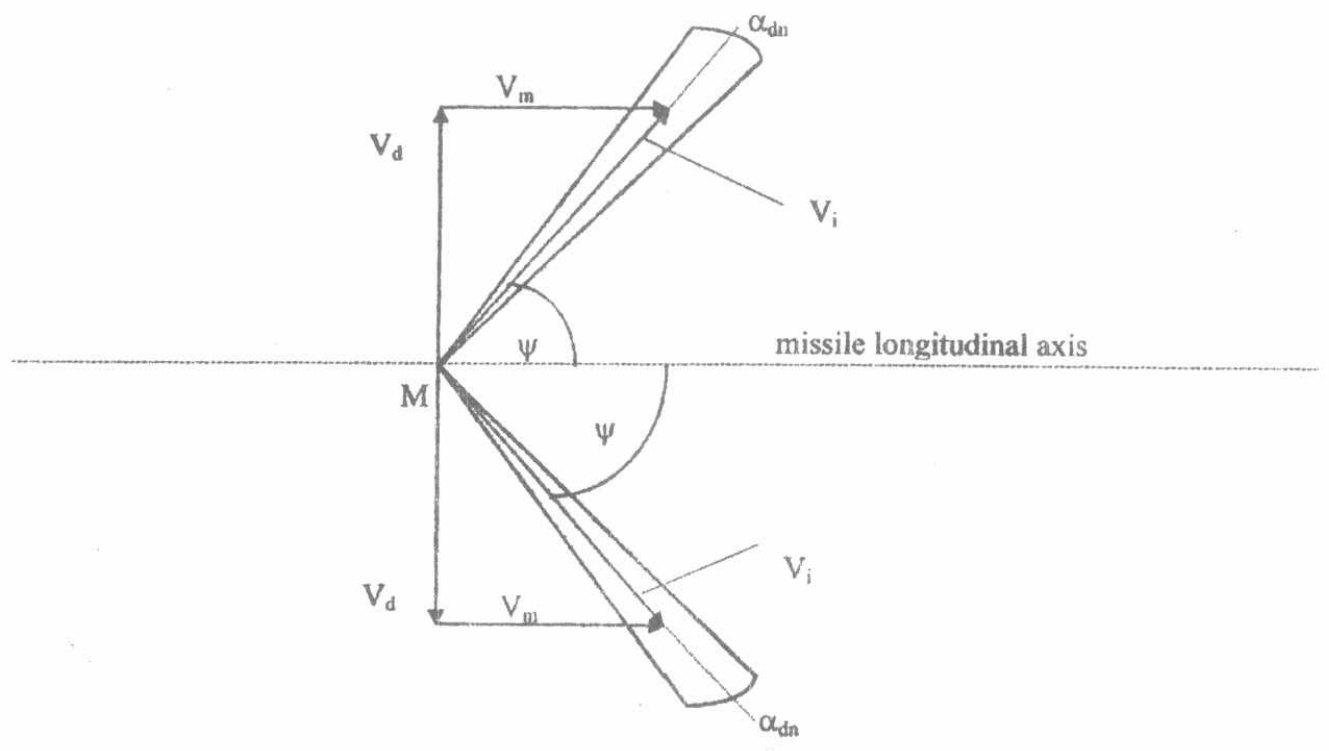

Fig. 1: Dynamic destruction zone 
e. Possible target destruction zone, the possible target destruction zone is the locus of target positions at the moment of warhead initiation securing the target hitting at least by one fragment. The optimum position of the target at the moment of the warhead initiation is on the central axis of the target destruction zone. If the missile velocity is directed along the missile longitudinal axis, parallel to the target velocity, and the missile and the target are approaching,

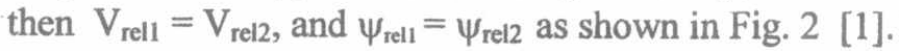

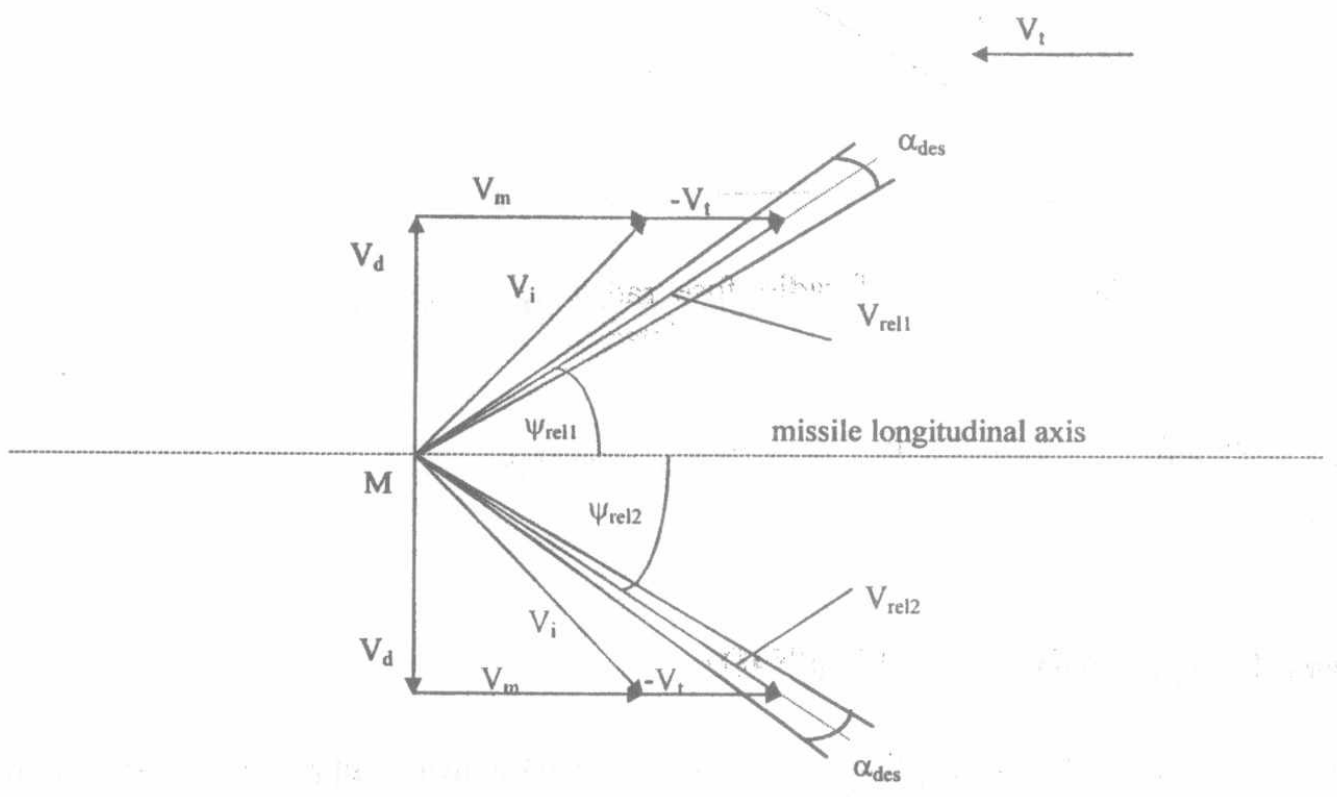

Fig. 2: Possible target destruction zone

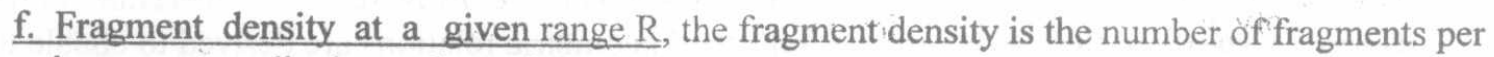
unit area perpendicular to the radial velocity $\underset{V_{i}}{\longrightarrow}$ and calculated by [1] as:

$$
F=\frac{\gamma_{n} N}{\left[2 \pi R\left(h+2 R \tan \left(\frac{\alpha_{s t}}{2}\right)\right)\right]}
$$

Where $\mathrm{F}$ is the fragment density at distance $\mathrm{R}$, and $\gamma_{n} N$ is the number of fragments within the zone of dispersion,

g. Radio fuse switching zone, The radio fuse-switching zone is a virtual zone in space, which is shifted from the radiation pattern due to the inertia time of the processing operations in the radio fuse system [1].

The presence time of the target in the fuse radiation zone should be greater than the radio fuse switching lag time, i.e. $t_{\text {signal }}>t_{\text {lag }}$.

Where $t_{\text {lag }}$ is the radio fuse switching lag time, and $t_{\text {signal }}$ is the presence time of the target in the fuse radiation zone. It is given by [1] as:

$t_{\text {signal }}=\frac{L_{e}+Z_{s}}{V_{r}}$

Where $L_{e}$ is the equivalent length of the target in the direction of the missile-target relative velocity, and $Z_{s}$ is the width of the radiation pattern at the determined value of the missiletarget missdistance as shown in Fig. 3 [2]. 


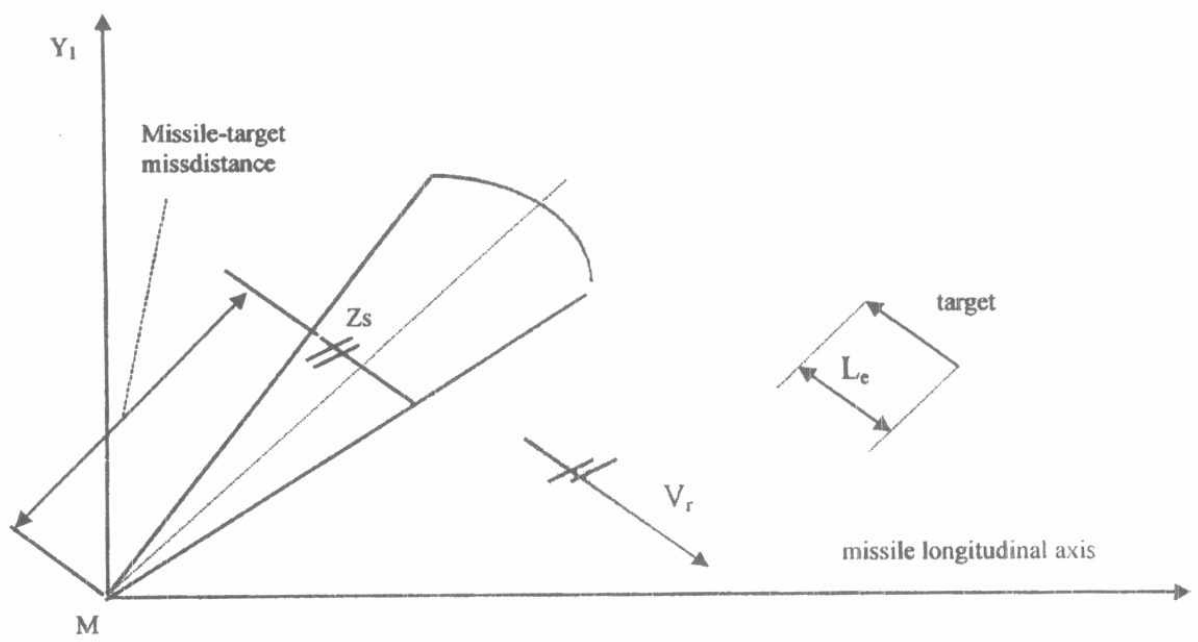

Fig. 3: Width of radio fuse radiation pattern at a specific missile-target miss distance

Then, the following condition should be achieved as [1]:

$Z s_{\min }>t_{\text {lag }} V_{r}-L_{e}$

\section{3-MATHEMATICAL MODEL}

The radio fuse and the warhead are described by many technical parameters. Some of these parameters are found to be best described as random parameters. One of such parameters is the static destruction zone subtended angle $\alpha_{\text {stat }}$, which varies from 5 to 30 degrees. A Gaussian distribution is considered, since it simulates closely the real operation of the involved system. In the following, the important geometric relations are derived to enable the evaluation of the radio fuse system. Fig. 4 shows the missile-target geometry at the explosion moment, where $V_{i}$ is the initial speed of the fragment, $V_{r}$ is the relative velocity between the missile and the target, and $V_{\text {rel }}$ is the relative velocity of the fragment with respect to target velocity.

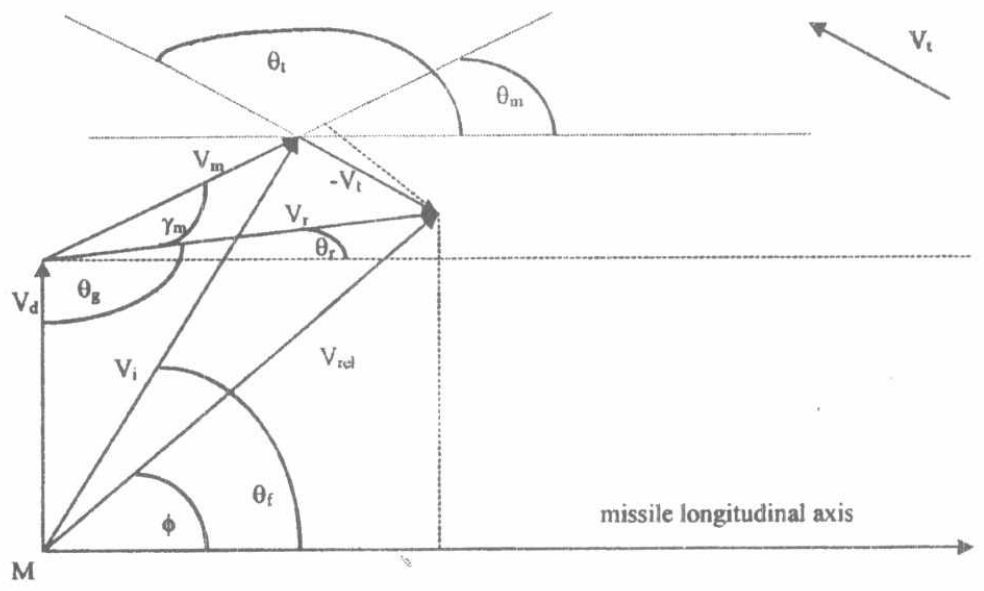

Fig. 4: Missile-Target geometry at the explosion moment 
Thus, as [5]:

$$
\begin{aligned}
& V_{i}=\sqrt{V_{d}^{2}+V_{m}^{2}+2 V_{d} V_{m} \sin \left(\theta_{m}\right)} \\
& \theta_{f}=\sin ^{-1}\left[\frac{V_{d}+V_{m} \sin \left(\theta_{m}\right)}{V_{i}}\right] \\
& V_{r}=\sqrt{V_{m}^{2}+V_{i}^{2}-2 V_{m} V_{t} \cos \left(\theta_{t}-\theta_{m}\right)} \\
& \theta_{r}=\theta_{m}-\sin ^{-1}\left[\frac{V_{t} \sin \left(\theta_{t}-\theta_{m}\right)}{V_{r}}\right] \\
& \phi=\tan -1\left[\frac{V_{d}+V_{r} \sin \left(\theta_{r}\right)}{V_{r} \cos \left(\theta_{r}\right)}\right]
\end{aligned}
$$

The target locations from the detection instant to the intercept with fragment moment are shown in Fig. 5, where:

$\mathrm{T}$ is the target location at the detection moment,

$\mathrm{V}$ is the target location at the warhead destruction moment, $\mathrm{X}$ represents the future location of the target at the possible intercept position with the fragment, and $\varphi_{\mathrm{a}}$ is the target detection angle by the radio fuse antenna.

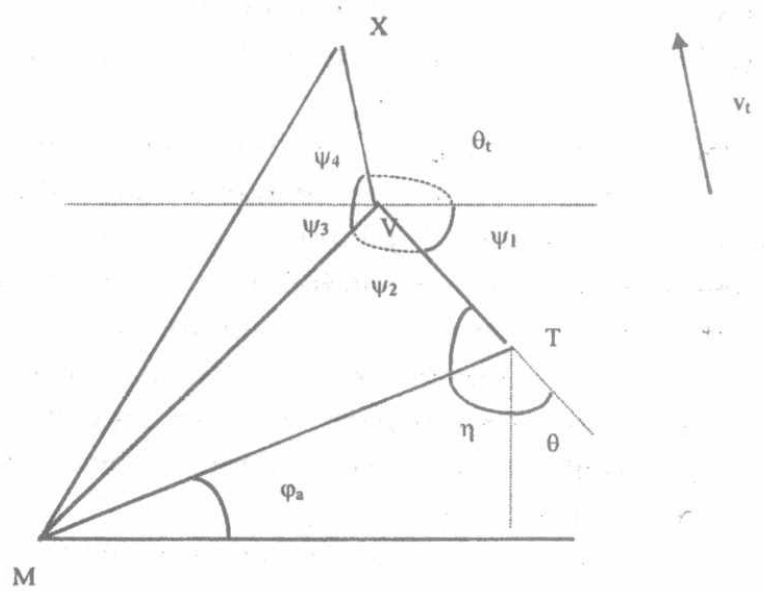

M

Fig. 5: Target locations from detection instant to possible intercept with fragment moment

Thus:

$T V=V_{r} d t$

Where dt is the delay time from the target detection moment to warhead explosion moment. Then, by [5]:

$$
\begin{aligned}
& M V=\sqrt{M T^{2}+T V^{2}-2(M T)(T V) \cos (M \hat{T} V)} \\
& V \hat{M} X=\left|\theta_{f}-\phi\right| \\
& \psi_{1}=90-\theta \\
& \psi_{2}=\sin ^{-1}\left[\frac{M T}{M V} \sin (M \hat{T} V)\right]
\end{aligned}
$$


$\psi_{4}=180-\theta_{t}$

$\psi_{3}=180-\left(\psi_{1}+\psi_{2}\right)$

$M \hat{V X}=\psi_{3}+\psi_{4}$

$M \hat{X} V=180-(M \hat{V} X+V \hat{M} X)$

$M X=\frac{2 \Delta_{M V X}}{(M V) \sin (V \hat{M} X)}$

Where $\Delta_{M V X}$ is the area of the triangular MVX,

Operation of the radio fuse system under consideration depends on the delay time principle from the target detection instant to the warhead explosion moment. This time is required for charging a capacitor in the decision circuit to a sufficient level to ignite the warhead.

\section{4-COMPUTATIONAL ALGORITHM}

A numerical algorithm is developed for evaluating the radio fuse performance. The algorithm considers both the geometrical engagement scenario (which is changing) and the fusing and arming system parameters. It can be summarized as follows:

1. The relative velocity between the missile and target $\mathrm{V}_{\mathrm{r}}$ is calculated from the geometrical considerations.

2. The target position relative to the missile at the explosion moment is calculated.

3. Initially, the number of fragments that hit the target is assumed zero. The initial width of the target destruction zone is considered equal the lower limit value. The central axis of the destruction zone is determined. The nearest and farthest limit angles are thus calculated.

4.If the target passes through the detection zone prior to the destruction zone, and then warhead fragments may hit it. Otherwise, the number of fragments that may hit the target equals zero due to the impossible interception between the target and fragments.

5. The distances from the missile to the target locations at the detection instant and at the possible interception position are calculated.

6. The coincidence of the target area with the destruction zone is then calculated.

7. The distance from the missile to the new target location that corresponds to the interception with the warhead fragments is calculated.

8. The delay time from the target detection moment to the warhead explosion morment is determined from system considerations.

9. The calculations are repeated every incremental static destruction zone width $\mathrm{da}_{\mathrm{s}}$ and the instantaneous number of fragments, $n$ that hit the target area are weighted and summed up.

Fig. 6 shows a flow chart of the computational algorithm.

\section{5-NUMERICAL RESULTS}

An engagement scenario given by $V_{m}=1000 \mathrm{~m} / \mathrm{sec}, V_{t}=300 \mathrm{~m} / \mathrm{sec}, V_{d}=3000 \mathrm{~m} / \mathrm{sec}, \theta_{m}=0^{\circ}$ (missile incidence angle $=0^{\circ}$ ), missile speed is parallel to target speed, and a target length given by $5 \mathrm{~m}$, and width $=2 \mathrm{~m}$ are considered. The effect of terminal miss-distance on the number of 
fragments that may hit the target will be considered first. Fig. 7 shows this effect in the approaching and receding cases. It is clear that, the increase in the terminal miss distance for receding and approaching target cases sharply decreases the number of fragments $(\mathbb{N})$ that may hit the target; and hence the probability of kill. The decrease in the fragments number $\mathrm{N}$ is attributed to the decrease in the fragments spatial densify inside the fragments cones as the miss-distance increases. This result speculates the impottance of employing accurate guidance and control system in the improvement of the target kill probability.

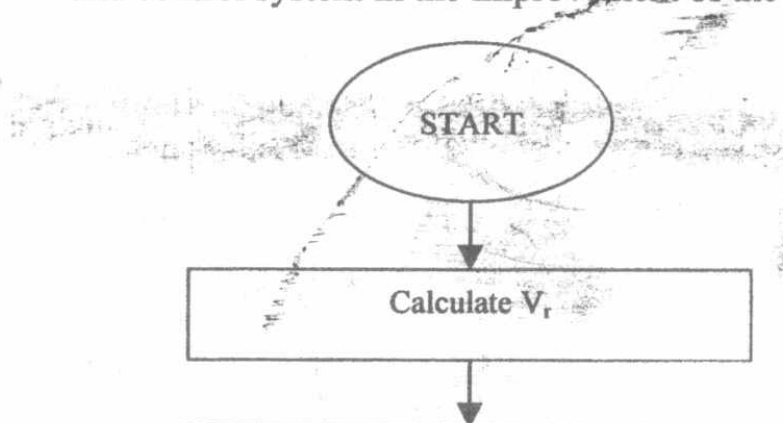

Calculate target position at the explosion moment

Calculate the generated number of fragments $\mathrm{N}$, and number of fragments that hit the target $\mathrm{N}_{\mathrm{h}}$,

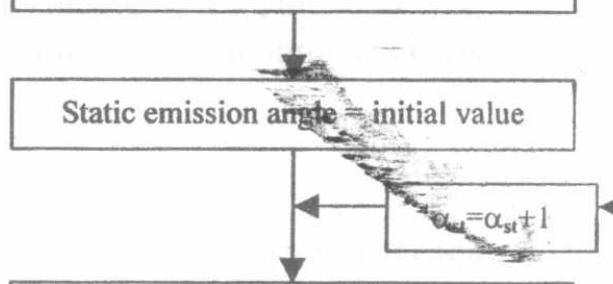

Determine destruction zone central axis angle $\phi$ and its nearest and farthest limits to the target $\phi_{1}$ and $\phi_{2}$

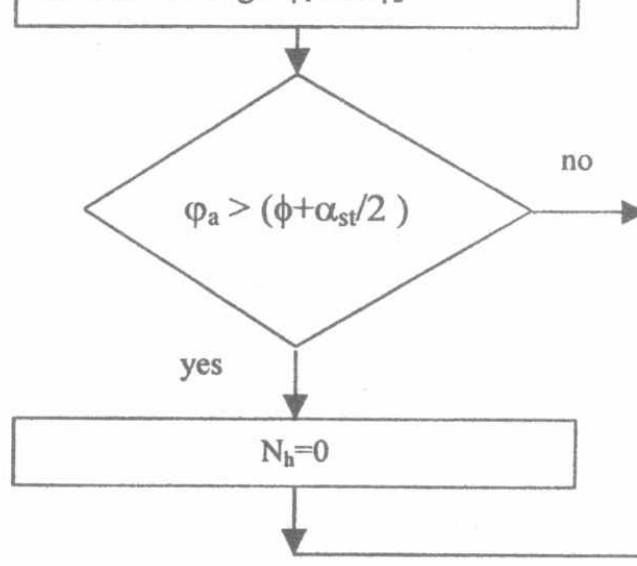

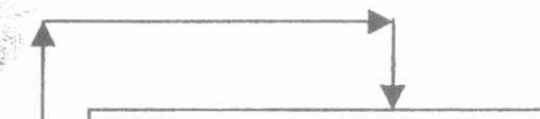

-Determine distance from missile to target positions at detection instant and target intersecting positions with central axis and nearest and farthest limits of the target destruction zone.

- Determine the distance from missile to the future target position that corresponds to the possible fragment collifsion.

- Catculate the delay time from target detection instant to warhead explosion instant $\mathrm{dt}$

- Calculate the number of fragments that may hit the target for the given static emission angle.

- Accumulate the number of fragments that may hit the target

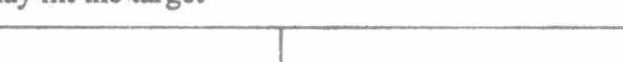

Fig. 6: Flow chart for numerical computation of the number of fragments that may hit the target for a given engagement scenario case. 


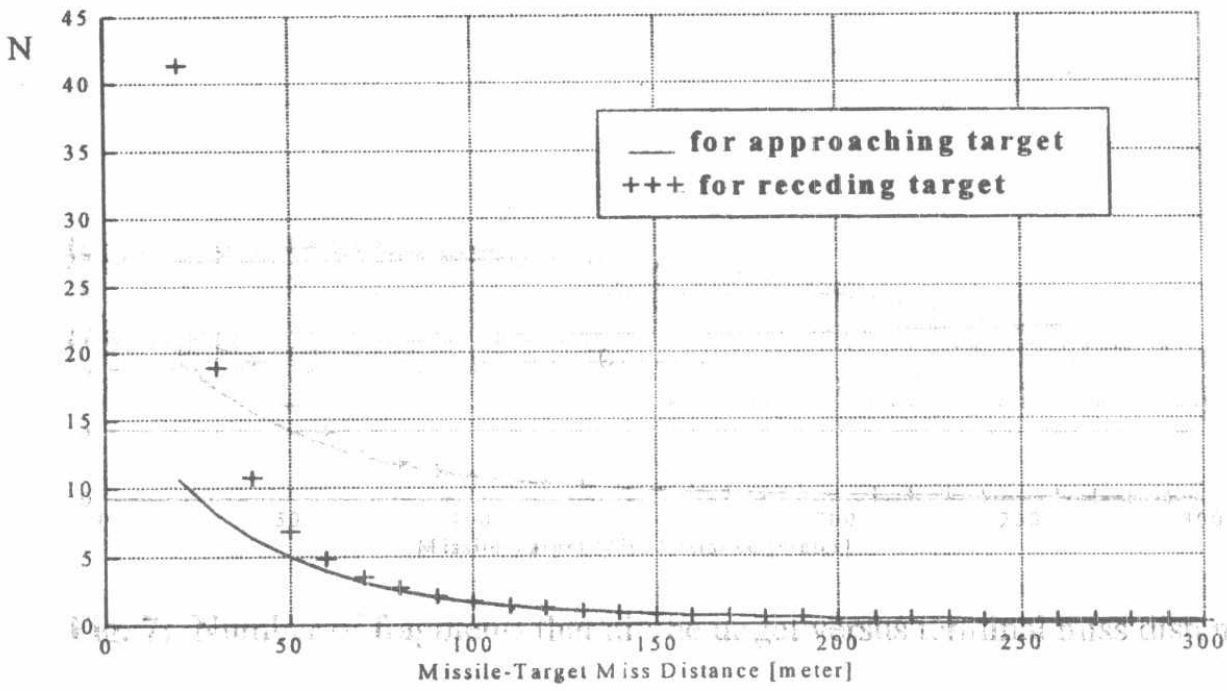

Fig. 7: Number of fragments that hit the farget versus terminal miss distance h

Fig. 8 shows the effect of target velocity on the number of fragments that may hit the target in the receding and approaching cases. The terminal miss distant is kept constant and is chosen by 70 meters. Other engagement parameters are as given before. It is clear that as the target velocity increases in the receding and approaching cases, the number of fragments that may hit the target decreases. This is due to the decrease of target stay time in the dynamic destruction zone.

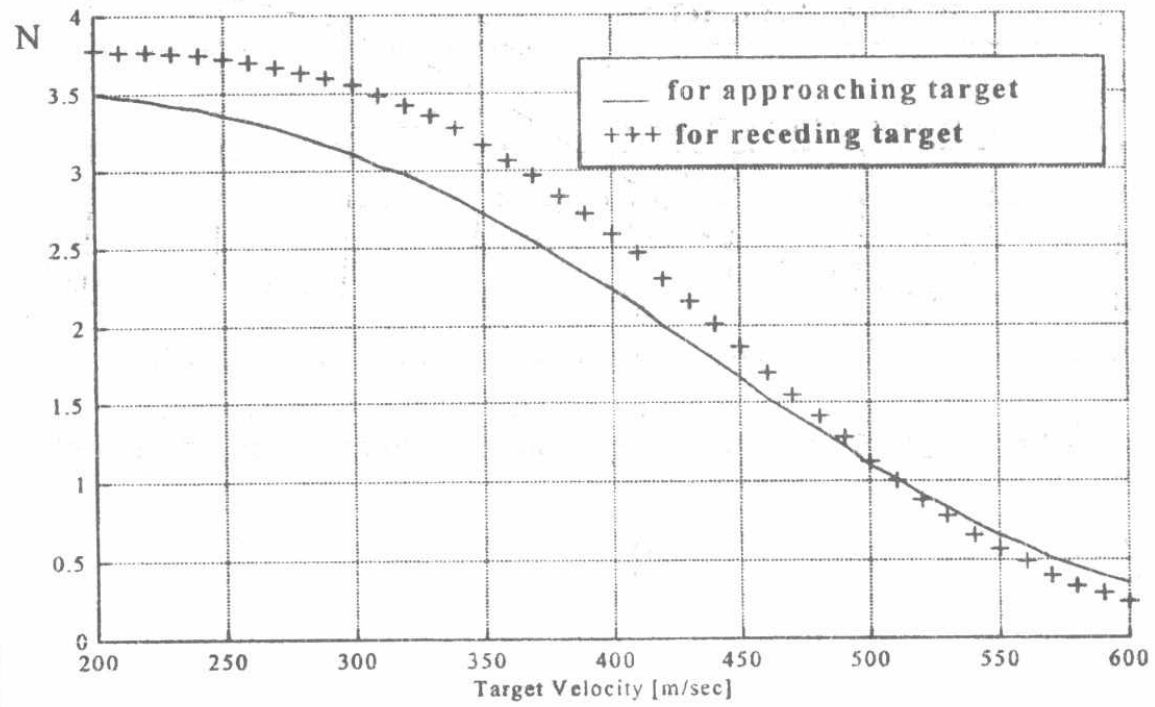

Fig. 8: Number of fragments that hit the target versus target velocity

$$
x^{2}
$$

On the other hand, the increase of the missile velocity implies that receding targets become closer to the dynamic destruction zone. So the number of fragments that may hit the target 
increases. Approaching targets go away from the dynamic destruction zone as the missile velocity increase as shown in Figs. 9-a, and 9-b.

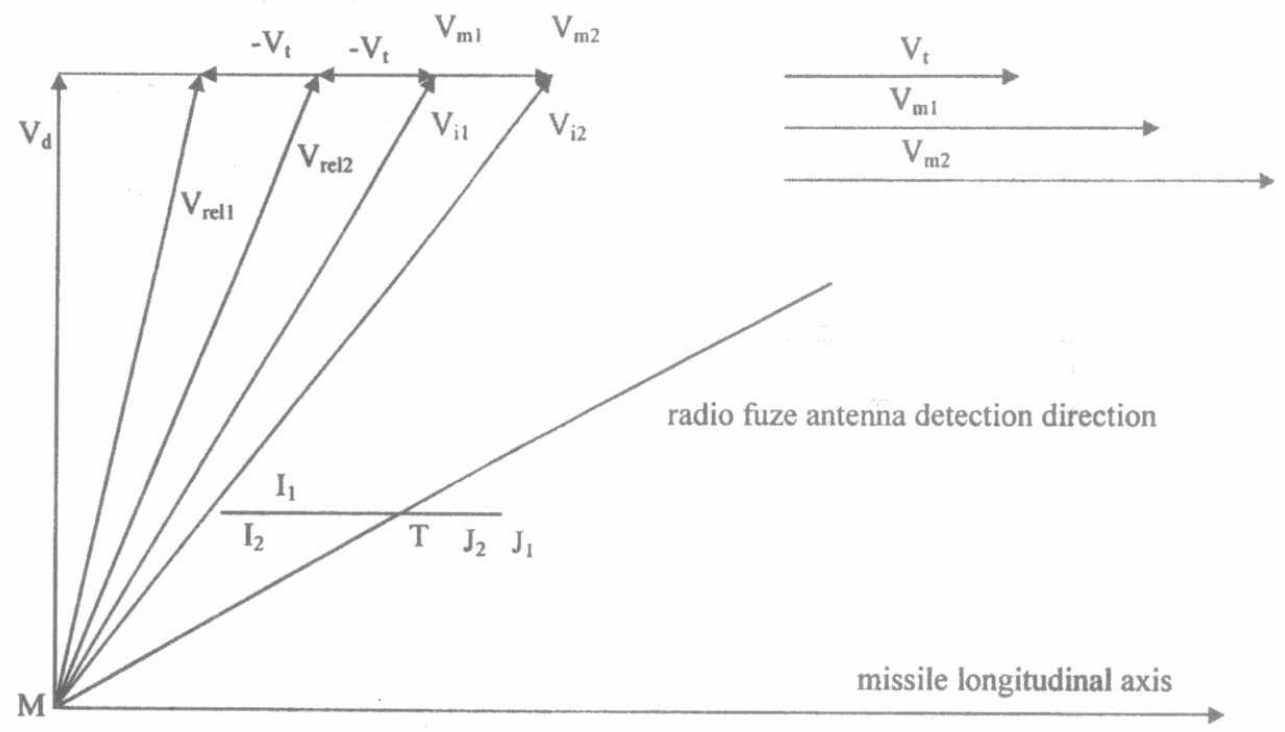

Fig. 9-a: Effect of missile velocity on missile-target engagement geometry at the warhead explosion moment in the receding case

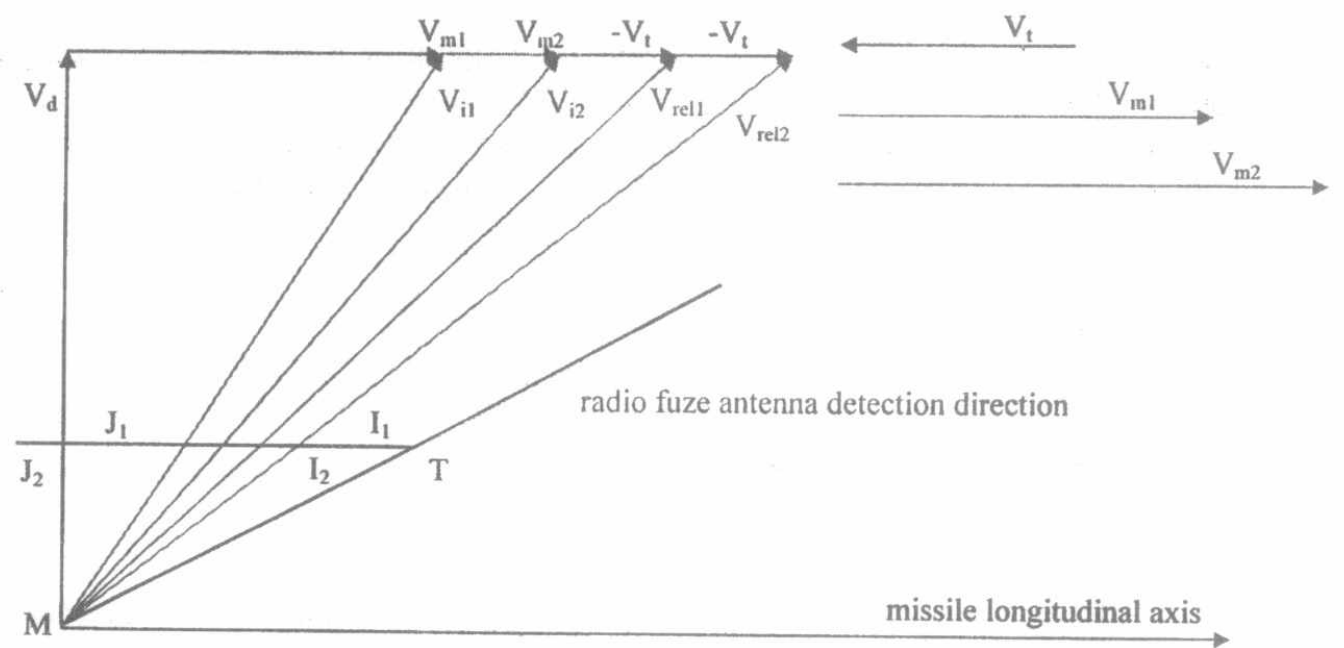

Fig. 9-b: Effect of missile velocity on missile-target engagement geometry at the warhead explosion moment in the approaching case

Where $I_{1}, I_{2}$ are the target positions at the warhead destuction moment, and $J_{1}, J_{2}$ are the future target positions at the possible intercept position with the fragment, Fig. 10 shows the effect of missile velocity on the number of fragments that may hit the target in the receding and approaching cases with terminal miss distant being kept constant chosen 70 meter and engagement parameters as given before. 


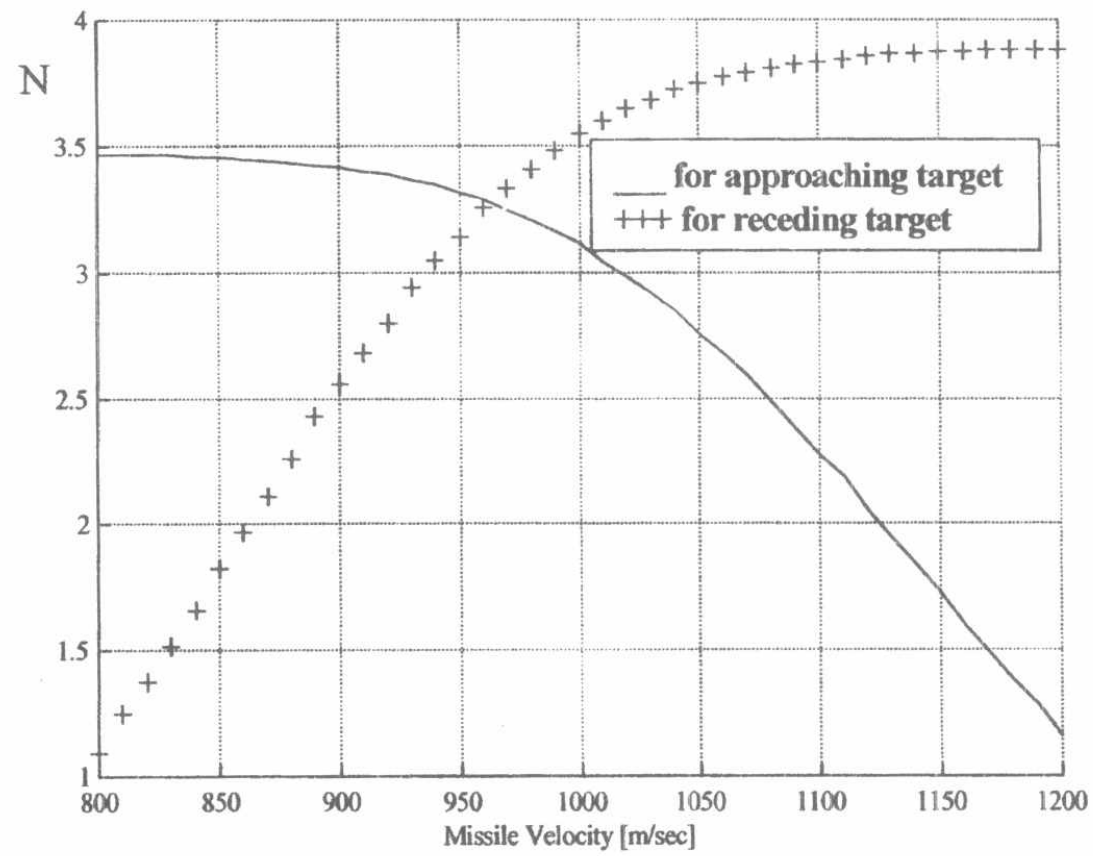

Fig. 10: Number of fragments that hit the target versus missile velocity

Fig. 11-a shows that for receding targets, the target goes away from the dynamic destruction zone by the increase of the fragment speed, so the number of fragments that may hit the target decreases. But for approaching targets, the target becomes closer to the dynamic destruction zone by the increase of the fragment speed as shown from Fig. 11-b.

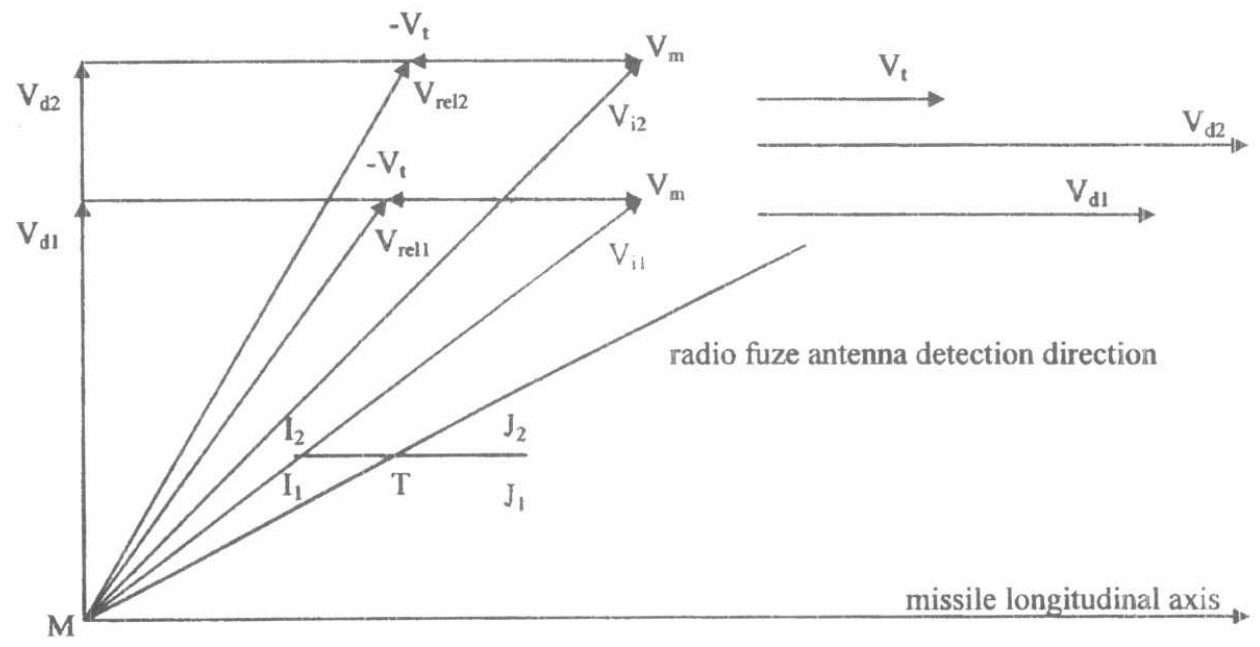

Fig. 11-a: Effect of fragment speed on missile-target engagement geometry at the warhead explosion moment for receding targets 


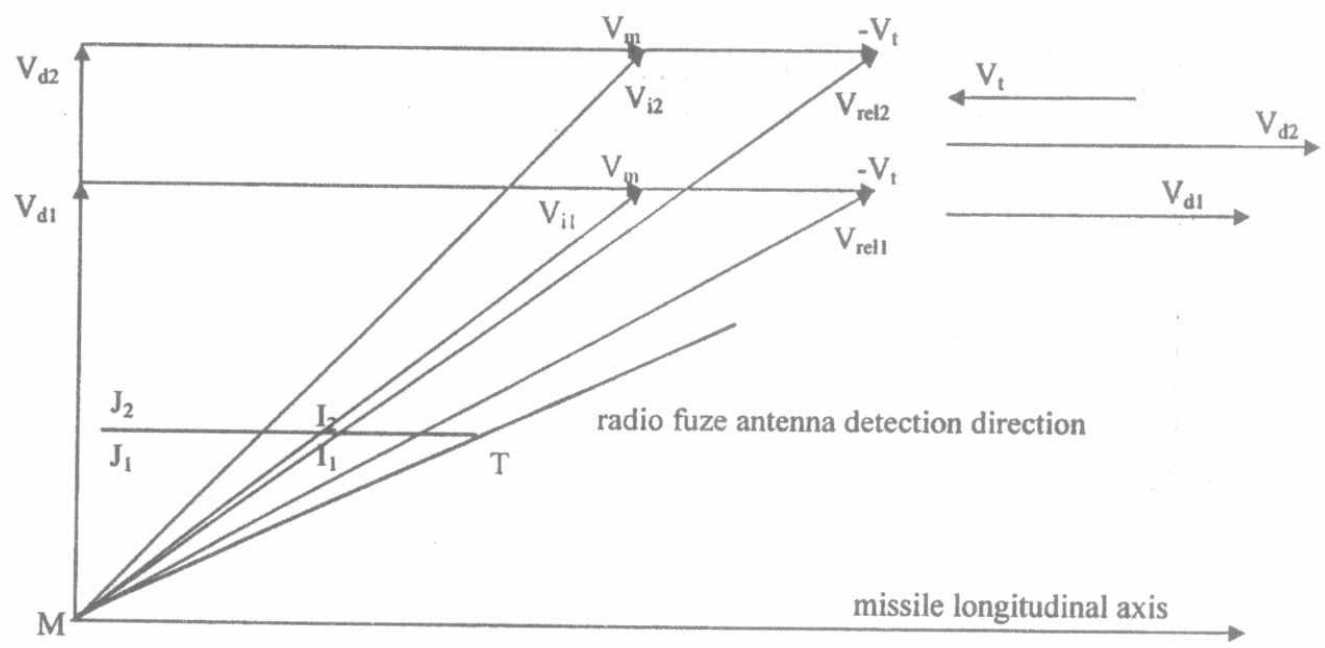

Fig. 11-b: Effect of fragment speed on missile-target engagement geometry at warhead explosion moment for approaching targets

Fig. 12 shows the effect of fragment speed on the number of fragments that may hit the target in the receding and approaching cases with terminal miss distant chosen by 70 meter and engagement parameters as given before.

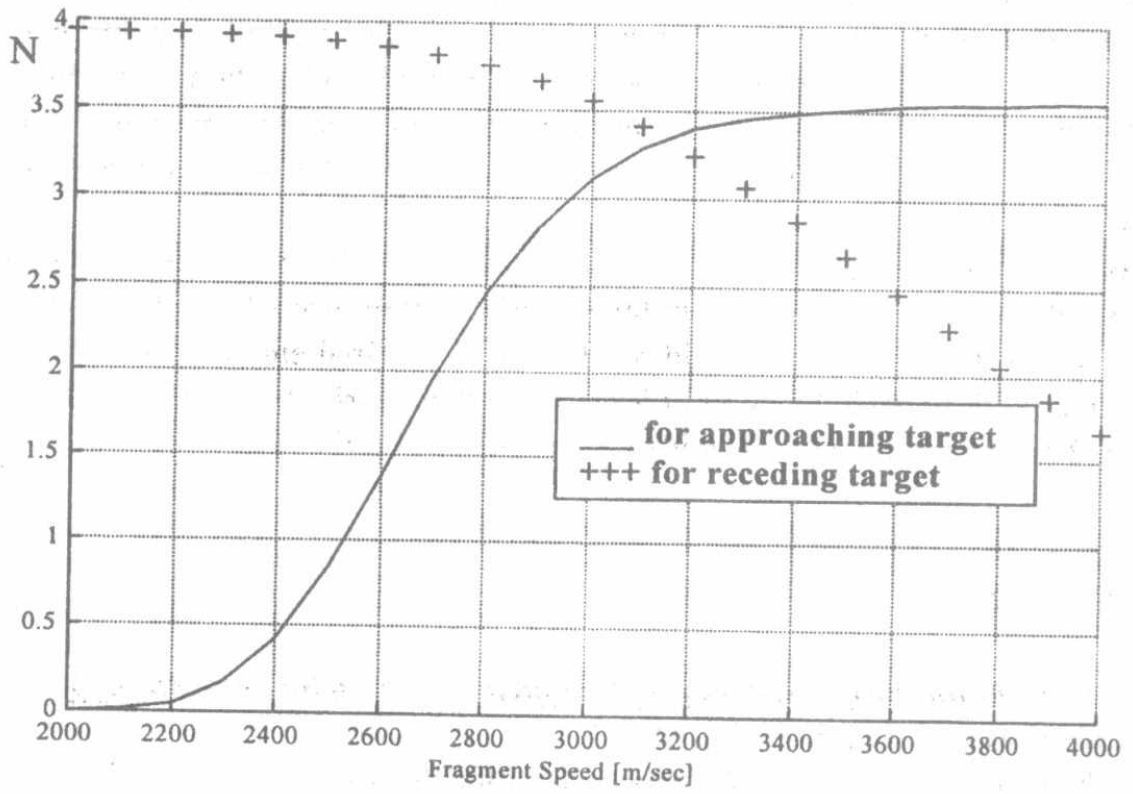

Fig. 12: Number of fragments that hit the target versus fragment speed

Finally, Fig. 13 shows that, the increase of the target length for receding and approaching targets increases the number of fragments that may hit the target. 


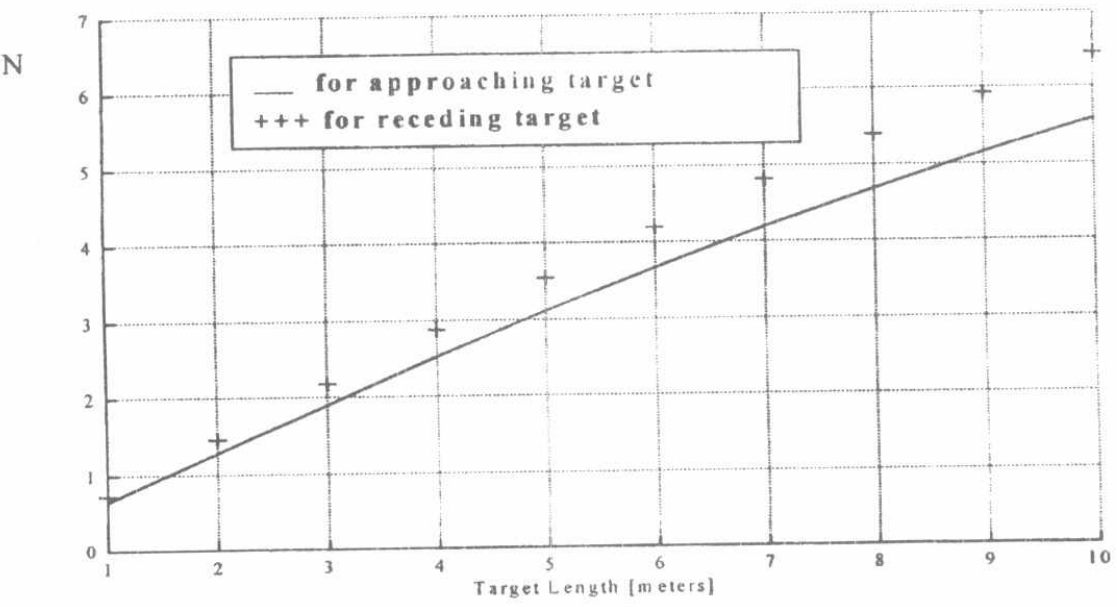

Fig. 13: Number of fragments that hit the target versus target length

It is clear that as the target length increases, higher number of fragments may hit the target.

\section{6-CONCLUSIONS}

In this paper, A computational algorithm is developed for computing the number of fragments that may hit the target that can be achieved via radio-fuse system. A computer code written with Borland $\mathrm{C}$ is built to solve the developed model and obtain numerical results. The number of fragments that may hit the engaged target is considered a measure for probability of target destruction. Thus, the number of fragments that hit the target is calculated for various engagement geometries.

The results show that the guidance and control system has to be properly designed so that the terminal target miss is always within the effective range of warhead destruction zone. Also, the employed guidance method should result in optimal final engagement scenario that yields maximum destruction probability or at least defined scenario. Upon this defined scenario, the fuse system should be tailored to provide maximum destruction probability.

\section{REFERENCES}

[1]Dusek Josef, Radio fuse of anti-aircraft guided rocket taking advantage of Doppler effect. Printed lectures TSR 179 S, M.T.C, 1970.

[2]Otakar Dvorak, Theory of radio fuses for anti-aircraft rockets, Printed lectures 776,

M.T.C, 1967.

[3] Jiri Kuchar, Rockets warheads, Printed lectures 786, M.T.C, 1967.

[4] A.EL.Monem.Saleh, Optimization of fuzing action for proximity fusing of anti-aircraft guided missiles, M.SC thesis, M.T.C, 1995.

[5] Jan J.Tuma, Engineering mathematics handbook, 1986. 